\title{
Elastic Multi-layered Analysis Using DE-Integration
}

\author{
By \\ James Maina and Kunihito Matsui*
}

\section{$\S 1 . \quad$ Introduction}

In classical mechanics, solutions to various solid mechanics problems have very well been established. The most famous one is the Boussinesq solution for the case of concentrated vertical load acting on the surface of a semi-infinite body. However, in engineering problems like foundation, road or airport pavement design, solutions to a multi-layered system are required. Furthermore, simple but accurate and flexible solution approach which can be extended to multi-layered systems with various boundary conditions is of great necessity for engineering design.

Authors of this paper have already analyzed various types of loads acting simultaneously or separately on the surface of the pavement structure. A pavement is modeled as a multi-layered elastic structure on the surface of which circular wheel loads act. In general, a wheel load is assumed to exert a vertical surface load due to the weight of the vehicle while braking or accelerating the vehicle would exert horizontal surface load [1]. Moreover, when large-sized vehicles like trucks and trailers turn on sharp corners wheels may exert turning (torsional) load on the pavement surface [2]. Finally, a rolling or stationary tire on the surface of a pavement system generates not only vertical load but also centripetal shear load due to the fact that the wheel can not freely expand due to surface frictional force [3]. This centripetal shear load causes an increase in tensile stress at the surface of a pavement structure.

Theoretical analysis utilizes the principle of superposition and the solution is given by summing up the solution of the various loads considered. In the

Communicated by H. Okamoto. Received December 10, 2004. Revised March 30, 2005. 2000 Mathematics Subject Classification(s): 74B05

${ }^{*}$ College of Science and Engineering, Tokyo Denki University, Saitama 350-0394, Japan. 
analytical system developed by the authors, 10,000 measurement points can be analyzed using a maximum of 100 loads and 100 pavement layers. This will enable engineers determine stresses, displacements and strains at any point within the pavement structure. It is considered that accurate analysis of the effects of these different loads may contribute to the improvements of methods for design of new pavements or evaluation of existing pavements for maintenance or rehabilitation purposes and hence significantly reduce the cost involved thereof.

Since numerical analysis of these loads showed similar characteristics, authors decided to present, in this paper, a very basic solution due to vertical loading that makes use of the Hankel transform. In this research, double exponential (DE) formula developed by Prof. Mori was utilized to facilitate semi-infinite numerical integration [4] and the Fortran program available at Dr. Ooura's webpage was used. A worked example for one layer system is presented in order to check accuracy of DE-integration against the prescribed boundary conditions. Problems with the implementation of numerical integration were found only in the vicinity of the pavement surface i.e. $z=0$.

\section{$\S 2 . \quad$ Problem Formulation}

Consider a multi-layered structure shown in Figure 1, on the surface of which a uniformly distributed circular load is acting in the vertical direction. The arrows on the infinitesimal block indicate positive direction of the stresses. Equations of the theory of elasticity for three-dimensional problem in cylindrical coordinates, which satisfy equilibrium and compatibility conditions, are employed.

\section{$\S 2.1$. Equations of equilibrium}

The following two equations represent equilibrium condition of the infinitesimal block shown in Figure 1.

$$
\begin{aligned}
& \frac{\partial \sigma_{r}}{\partial r}+\frac{\partial \tau_{r z}}{\partial z}+\frac{\sigma_{r}-\sigma_{\theta}}{r}=0 \\
& \frac{\partial \tau_{r z}}{\partial r}+\frac{\partial \sigma_{z}}{\partial z}+\frac{\tau_{r z}}{r}=0 .
\end{aligned}
$$




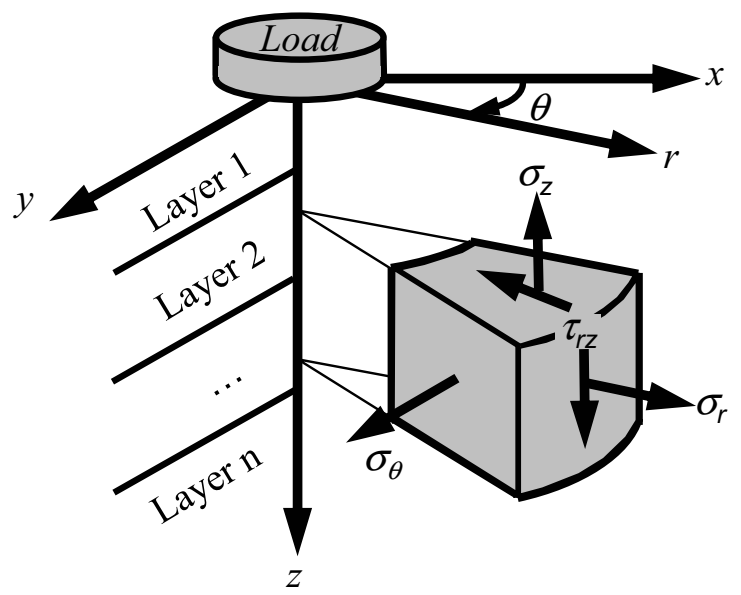

Figure 1. Multi-layered system

\section{§2.2. Stress-displacement relationship from Hooke's law}

The relation between stress and displacement can be represented in the following manner:

$$
\begin{aligned}
\sigma_{r} & =\lambda\left(\frac{\partial u}{\partial r}+\frac{u}{r}+\frac{\partial w}{\partial z}\right)+2 \mu \frac{\partial u}{\partial r}, \\
\sigma_{\theta} & =\lambda\left(\frac{\partial u}{\partial r}+\frac{u}{r}+\frac{\partial w}{\partial z}\right)+2 \mu \frac{u}{r}, \\
\sigma_{z} & =\lambda\left(\frac{\partial u}{\partial r}+\frac{u}{r}+\frac{\partial w}{\partial z}\right)+2 \mu \frac{\partial w}{\partial z}, \\
\tau_{r z} & =\mu\left(\frac{\partial u}{\partial z}+\frac{\partial w}{\partial r}\right),
\end{aligned}
$$

where, $\lambda=\frac{\nu E}{(1+\nu)(1-2 \nu)}, \mu=\frac{E}{2(1+\nu)}$.

Furthermore, $\left(\sigma_{r}, \sigma_{\theta}, \sigma_{z}\right)$ are normal stress components in the $(r, \theta, z)$ direction of the cylindrical coordinate system and $\tau_{r z}$ is the shearing stress on $r$-plane in the $z$-direction, which is similar to the shearing stress on $z$ plane in the $r$ direction of the cylindrical coordinate system. 


\section{$\S 2.3$. Elasticity equations of displacement}

Displacements can be expressed in terms of strain function, $\Phi$, in the following manner:

$$
\begin{aligned}
& u_{r}=-\frac{\partial^{2} \Phi}{\partial r \partial z}, \\
& u_{z}=2(1-\nu) \nabla^{2} \Phi-\frac{\partial^{2} \Phi}{\partial z^{2}},
\end{aligned}
$$

where, $u_{r}, u_{z}$ are displacement components in $(r, z)$-directions of the cylindrical coordinate system.

\section{§2.4. Elasticity equations of stress}

Substituting equations $(7)$ and $(8)$ into equations $(3) \sim(6)$, elasticity equations of stress may be expressed in terms of $\Phi$ as follows:

$$
\begin{aligned}
\frac{\sigma_{r}}{2 \mu} & =\frac{\partial}{\partial z}\left(\nu \nabla^{2}-\frac{\partial^{2}}{\partial r^{2}}\right) \Phi, \\
\frac{\sigma_{\theta}}{2 \mu} & =\frac{\partial}{\partial z}\left(\nu \nabla^{2}-\frac{1}{r} \frac{\partial}{\partial r}\right) \Phi, \\
\frac{\sigma_{z}}{2 \mu} & =\frac{\partial}{\partial z}\left((2-\nu) \nabla^{2}-\frac{\partial^{2}}{\partial z^{2}}\right) \Phi, \\
\frac{\tau_{r z}}{2 \mu} & =\frac{\partial}{\partial r}\left((1-\nu) \nabla^{2}-\frac{\partial^{2}}{\partial z^{2}}\right) \Phi,
\end{aligned}
$$

where, $\nu$ is Poisson's ratio.

\section{$\S 2.5$. Equations of compatibility}

The compatibility equation is expressed as follows:

$$
\nabla^{4} \Phi=0
$$

where

$$
\nabla^{2}=\frac{\partial^{2}}{\partial r^{2}}+\frac{1}{r} \frac{\partial}{\partial r}+\frac{\partial^{2}}{\partial z^{2}} .
$$

$\nabla^{2}$ is the axi-symmetric Laplace operator and the stress function, $\Phi$, is a solution of the biharmonic equation. 


\section{§3. The Hankel Transform of the Stress Function}

Performing the Hankel transform [5] on compatibility equation yields:

$$
\int_{0}^{\infty} r \nabla^{4} \Phi J_{0}(\xi r) d r=\left(\frac{d^{2}}{d z^{2}}-\xi^{2}\right)^{2} \int_{0}^{\infty} r \Phi J_{0}(\xi r) d r=0,
$$

where, $J_{0}(\xi r)$ is the Bessel function of the first kind of order zero. Let $G(\xi, z)=$ $\int_{0}^{\infty} r \Phi J_{0}(\xi r) d r$, and substituting into equation (15) yields:

$$
\left(\frac{d}{d z^{2}}-\xi^{2}\right)^{2} G(\xi, z)=0
$$

In order for equation (16) to be satisfied, $G(\xi, z)$ should be:

$$
G(\xi, z)=(A+B z) e^{-\xi z}+(C+D z) e^{\xi z} .
$$

Furthermore, differentiating $G(\xi, z)$ with respect to $z$ gives:

$$
\begin{aligned}
\frac{d G}{d z} & =-\xi e^{-\xi z} A+(1-\xi z) e^{-\xi z} B+\xi e^{\xi z} C+(1+\xi z) e^{\xi z} D \\
\frac{d^{2} G}{d z^{2}} & =\xi^{2} e^{-\xi z} A+(-2+\xi z) \xi e^{-\xi z} B+\xi^{2} e^{\xi z} C+(2+\xi z) \xi e^{\xi z} D \\
\frac{d^{3} G}{d z^{3}} & =-\xi^{3} e^{-\xi z} A+(3-\xi z) \xi^{2} e^{-\xi z} B+\xi^{3} e^{\xi z} C+(3+\xi z) \xi^{2} e^{\xi z} D
\end{aligned}
$$

\section{$\S 4$. Derivation of Displacement and Stress Using} the Hankel Transform

The Hankel transforms $\bar{u}_{r}, \bar{u}_{z}, \bar{\sigma}_{z}$, and $\bar{\sigma}_{z}$ are computed as follows:

$$
\begin{aligned}
\bar{u}_{r}= & \xi \frac{\partial}{\partial z} \int_{0}^{\infty} r \Phi J_{0}(\xi r) d r \\
= & \xi \frac{d G}{d z} \\
= & -\xi^{2} e^{-\xi z} A+(1-\xi z) \xi e^{-\xi z} B+\xi^{2} e^{\xi z} C+(1+\xi z) \xi e^{\xi z} D \\
\bar{u}_{z}= & 2(1-\nu)\left[\frac{d^{2}}{d z^{2}}-\xi^{2}\right] G-\frac{d^{2} G}{d z^{2}} \\
= & (1-2 \nu) \frac{d^{2} G}{d z^{2}}-2(1-\nu) \xi^{2} G \\
= & -\xi^{2} e^{-\xi z} A+(-2+4 \nu-\xi z) \xi e^{-\xi z} B \\
& \quad-\xi^{2} e^{\xi z} C+(2-4 \nu-\xi z) \xi e^{\xi z} D
\end{aligned}
$$




$$
\begin{aligned}
\bar{\sigma}_{z}= & 2 \mu\left[(1-\nu) \frac{d^{3} G}{d z^{3}}-(2-\nu) \xi^{2} \frac{d G}{d z}\right] \\
= & 2 \mu\left[\xi^{3} e^{-\xi z} A+(1-2 \nu+\xi z) \xi^{2} e^{-\xi z} B\right. \\
& \left.-\xi^{3} e^{\xi z} C+(1-2 \nu-\xi z) \xi^{2} e^{\xi z} D\right], \\
\bar{\tau}_{r z}= & -2 \mu \xi \int_{0}^{\infty} r\left[(1-\nu) \nabla^{2} \Phi-\frac{\partial^{2} \Phi}{\partial z^{2}}\right] J_{0}(\xi r) d r \\
= & 2 \mu \xi\left[\nu \frac{d^{2} G}{d z^{2}}+(1-\nu) \xi^{2} G\right] \\
= & 2 \mu\left[\xi^{3} e^{-\xi z} A+(-2 \nu+\xi z) \xi^{2} e^{-\xi z} B\right. \\
& \left.+\xi^{3} e^{\xi z} C+(2 \nu+\xi z) \xi^{2} e^{\xi z} D\right] .
\end{aligned}
$$

Derivation of the Hankel transforms of $\sigma_{r}$ and $\sigma_{\theta}$ is not straight forward but can be performed in the following manner:

$$
\begin{aligned}
H_{1}= & \int_{0}^{\infty} r\left\{\sigma_{r}+\frac{2 \mu u_{r}}{r}\right\} J_{0}(\xi r) d r \\
= & \int_{0}^{\infty} r\left\{\frac{\partial}{\partial z}\left[(\nu-1) \nabla^{2} \Phi+\frac{\partial^{2} \Phi}{\partial z^{2}}\right]\right\} J_{0}(\xi r) d r \\
= & \nu \frac{d^{3} G}{d z^{3}}+(1-\nu) \xi^{2} \frac{d G}{d z} \\
= & -\xi^{3} e^{-\xi z} A+(1+2 \nu-\xi z) \xi^{2} e^{-\xi z} B \\
& +\xi^{3} e^{\xi z} C+(1+2 \nu+\xi z) \xi^{2} e^{\xi z} D .
\end{aligned}
$$

Furthermore;

$$
\begin{aligned}
H_{2}= & \int_{0}^{\infty} r\left\{\sigma_{r}+\sigma_{\theta}\right\} J_{0}(\xi r) d r \\
= & \int_{0}^{\infty} r\left\{\frac{\partial}{\partial z}\left[(2 \nu-1) \nabla^{2} \Phi+\frac{\partial^{2} \Phi}{\partial z^{2}}\right]\right\} J_{0}(\xi r) d r \\
= & 2 \nu \frac{d^{3} G}{d z^{3}}+(1-2 \nu) \xi^{2} \frac{d G}{d z} \\
= & -\xi^{3} e^{-\xi z} A+(1+4 \nu-\xi z) \xi^{2} e^{-\xi z} B \\
& +\xi^{3} e^{\xi z} C+(1+4 \nu+\xi z) \xi^{2} e^{\xi z} D .
\end{aligned}
$$


Hankel transform of displacements and stresses derived above may be written in a matrix form as follows:

$$
\left\{\begin{array}{c}
\bar{u}_{r}(\xi, z) \\
\bar{u}_{z}(\xi, z) \\
\bar{\sigma}_{z}(\xi, z) \\
\bar{\tau}_{r z}(\xi, z)
\end{array}\right\}=\left[P_{1}(\xi, z)\right]\left\{\begin{array}{l}
A(\xi) \\
B(\xi) \\
C(\xi) \\
D(\xi)
\end{array}\right\},
$$

where

$$
\begin{aligned}
& {\left[P_{1}(\xi, z)\right]=} \\
& {\left[\begin{array}{cccc}
-\xi^{2} e^{-\xi z} & \xi(1-\xi z) e^{-\xi z} & \xi^{2} e^{\xi z} & \xi(1+\xi z) e^{\xi z} \\
-\xi^{2} e^{-\xi z} & \xi(-2+4 \nu-\xi z) e^{-\xi z} & -\xi^{2} e^{\xi z} & \xi(2-4 \nu-\xi z) e^{\xi z} \\
2 \mu \xi^{3} e^{-\xi z} & 2 \mu \xi^{2}(1-2 \nu+\xi z) e^{-\xi z} & -2 \mu \xi^{3} e^{\xi z} & 2 \mu \xi^{2}(1-2 \nu-\xi z) e^{\xi z} \\
2 \mu \xi^{3} e^{-\xi z} & 2 \mu \xi^{2}(-2 \nu+\xi z) e^{-\xi z} & 2 \mu \xi^{3} e^{\xi z} & 2 \mu \xi^{2}(2 \nu-\xi z) e^{\xi z}
\end{array}\right] .}
\end{aligned}
$$

Furthermore, the Hankel transform for the remaining stresses, i.e. $\sigma_{r}$ and $\sigma_{\theta}$ may also be written in the following manner:

$$
\left\{\begin{array}{l}
H_{1}(\xi, z) \\
H_{2}(\xi, z)
\end{array}\right\}=\left[P_{2}(\xi, z)\right]\left\{\begin{array}{l}
A(\xi) \\
B(\xi) \\
C(\xi) \\
D(\xi)
\end{array}\right\}
$$

where

$$
\begin{aligned}
& {\left[P_{2}(\xi, z)\right]=} \\
& {\left[\begin{array}{llll}
-2 \mu \xi^{3} e^{-\xi z} & 2 \mu(1+2 \nu-\xi z) \xi^{2} e^{-\xi z} & 2 \mu \xi^{3} e^{\xi z} & 2 \mu(1+2 \nu+\xi z) \xi^{2} e^{\xi z} \\
-2 \mu \xi^{3} e^{-\xi z} & 2 \mu(1+4 \nu-\xi z) \xi^{2} e^{-\xi z} & 2 \mu \xi^{3} e^{\xi z} & 2 \mu(1+4 \nu+\xi z) \xi^{2} e^{\xi z}
\end{array}\right] .}
\end{aligned}
$$

The above equations show that solutions to these problems may be obtained analytically. Coefficients of integration, $A, B, C$, and $D$, which may be different in each layer, are functions of Hankel parameter, $\xi$. These coefficients may be determined from the specified boundary conditions.

\section{$\S 5 . \quad$ Boundary Conditions}

\section{$\S 5.1 . \quad$ Surface, $z=0$}

Since a vertical uniformly distributed circular load is considered, the following two equations represent surface boundary conditions due to circular load 
of radius $a$ :

$$
\begin{aligned}
& \text { 1. } r \leq a \quad\left\{\begin{array}{l}
\sigma_{z}(r, 0)=-p, \\
\tau_{r z}=0,
\end{array}\right. \\
& \text { 2. } r>a \quad\left\{\begin{array}{l}
\sigma_{z}(r, 0)=0, \\
\tau_{r z}=0,
\end{array}\right.
\end{aligned}
$$

where, $\sigma_{z}$ and $\tau_{r z}$ are stress components as defined in Figure 1 and $p$ is the external uniformly distributed vertical load.

\section{§5.2. Between layers $i$ and $i+1$}

The following equations satisfy the equilibrium conditions at the layer interface:

$$
\begin{aligned}
u_{r}^{(i)}\left(r, h_{i}\right) & =u_{r}^{(i+1)}(r, 0), \\
u_{z}^{(i)}\left(r, h_{i}\right) & =u_{z}^{(i+1)}(r, 0), \\
\sigma_{z}^{(i)}\left(r, h_{i}\right) & =\sigma_{z}^{(i+1)}(r, 0), \\
\tau_{r z}^{(i)}\left(r, h_{i}\right) & =\tau_{r z}^{(i+1)}(r, 0),
\end{aligned}
$$

where, $r$ is the horizontal position in the cylindrical coordinate system and $h_{i}$ is the thickness of layer $i$.

\section{§5.3. Infinity, $z=\infty$}

The bottom layer is semi-infinite $\left(h_{n} \rightarrow \infty\right)$ and all responses (stress, displacement and strains) approach zero as $z$ approaches $\infty$.

These conditions are applicable to all layers in the multi-layered system. The Hankel transform considering boundary condition at the surface of the pavement as well as the uniformly distributed circular load, $p$, whose radius is $a$ would be given as:

$$
\left\{\begin{array}{c}
\bar{\sigma}_{z}^{1}(0, \xi) \\
\bar{\tau}_{r z}^{1}(0, \xi)
\end{array}\right\}=\left\{\begin{array}{c}
-\bar{p}(\xi) \\
0
\end{array}\right\},
$$

where the Hankel transform of the load, $\bar{p}$, may be determined as:

$$
\bar{p}(\xi)=\int_{0}^{\infty} r p J_{0}(\xi r) d r=\frac{p a}{\xi} J_{1}(\xi a) .
$$

By using equations (27) and (29), it is possible to develop a global propagation matrix showing the relationship between displacement and stress components of the $1^{\text {st }}$ layer and coefficients of integration of the lowest layer (the 
$N^{t h}$ layer). Furthermore, when $z \rightarrow \infty$ in case of the $N^{t h}$ layer (semi-infinite medium), displacement and stress components approach zero and coefficients of integration will become: $D_{n}=C_{n}=0$.

By taking into consideration boundary condition at the surface of the pavement where there is a uniformly distributed circular load, $p$, with radius $a$ as well as interface conditions and represent components of the global propagation matrix by $t_{i j}$, the relationship between the $1^{\text {st }}$ and $N^{t h}$ layers in the Hankel transform domain would be given as:

$$
\left\{\begin{array}{c}
\bar{u}_{r}^{1} \\
\bar{u}_{z}^{1} \\
\bar{\sigma}_{z}^{1} \\
\bar{\tau}_{r z}^{1}
\end{array}\right\}=\left\{\begin{array}{c}
\bar{u}_{r}^{1}(0, \xi) \\
\bar{u}_{z}^{1}(0, \xi) \\
-\bar{p} \\
0
\end{array}\right\}=\left[\begin{array}{cccc}
t_{11} & t_{12} & t_{13} & t_{14} \\
t_{21} & t_{22} & t_{23} & t_{24} \\
t_{31} & t_{32} & t_{33} & t_{34} \\
t_{41} & t_{42} & t_{43} & t_{44}
\end{array}\right]\left\{\begin{array}{c}
A_{n} \\
B_{n} \\
0 \\
0
\end{array}\right\},
$$

and from the above relation, it is found that coefficients of integration for the $N^{\text {th }}$ layer may be determined as:

$$
\left\{\begin{array}{l}
A_{n} \\
B_{n}
\end{array}\right\}=\left[\begin{array}{ll}
t_{31} & t_{32} \\
t_{41} & t_{42}
\end{array}\right]^{-1}\left\{\begin{array}{l}
\bar{p} \\
0
\end{array}\right\} .
$$

By using coefficients of integration for the $N^{\text {th }}$ layer, coefficients of integration $(A, B, C$, and $D)$ for other layers could be computed in a stepwise process.

\section{$\S 6 . \quad$ Solution}

After solving for coefficients of integration, it is possible to perform the inverse transform where responses at any point of interest may be determined:

\section{§6.1. The inverse Hankel transform}

The inverse Hankel transform of $\bar{u}_{r}, \bar{u}_{z}, \bar{\sigma}_{z}, \bar{\tau}_{r z}, \bar{\sigma}_{r}$ and $\bar{\sigma}_{\theta}$ may be determined, respectively as follows:

$$
\begin{aligned}
u_{r}= & \int_{0}^{\infty} \xi\left[-\xi^{2} e^{-\xi z} A+(1-\xi z) \xi e^{-\xi z} B\right. \\
& \left.+\xi^{2} e^{\xi z} C+(1+\xi z) \xi e^{\xi z} D\right] J_{1}(\xi r) d \xi, \\
u_{z}= & \int_{0}^{\infty} \xi\left[-\xi^{2} e^{-\xi z} A+(-2+4 \nu-\xi z) \xi e^{-\xi z} B\right. \\
& \left.-\xi^{2} e^{\xi z} C+(2-4 \nu-\xi z) \xi e^{\xi z} D\right] J_{0}(\xi r) d \xi,
\end{aligned}
$$




$$
\begin{aligned}
\sigma_{z}= & 2 \mu \int_{0}^{\infty} \xi\left[\xi^{3} e^{-\xi z} A+(1-2 \nu+\xi z) \xi^{2} e^{-\xi z} B\right. \\
& \left.-\xi^{3} e^{\xi z} C+(1-2 \nu-\xi z) \xi^{2} e^{\xi z} D\right] J_{0}(\xi r) d \xi, \\
\tau_{r z}= & 2 \mu \int_{0}^{\infty} \xi\left[\xi^{3} e^{-\xi z} A+(-2 \nu+\xi z) \xi^{2} e^{-\xi z} B\right. \\
& \left.+\xi^{3} e^{\xi z} C+(2 \nu+\xi z) \xi^{2} e^{\xi z} D\right] J_{1}(\xi r) d \xi \\
\sigma_{r}= & 2 \mu\left[\int_{0}^{\infty} \xi\left\{\frac{\bar{\sigma}_{r}}{2 \mu}+\frac{\bar{u}_{r}}{r}\right\} J_{0}(\xi r) d \xi-\frac{u_{r}}{r}\right] \\
= & 2 \mu \int_{0}^{\infty} \xi\left[-\xi^{3} e^{-\xi z} A+(1+2 \nu-\xi z) \xi^{2} e^{-\xi z} B\right. \\
& \left.+\xi^{3} e^{\xi z} C+(1+2 \nu+\xi z) \xi^{2} e^{\xi z} D\right] J_{0}(\xi r) d \xi \\
& -\frac{2 \mu}{r} \int_{0}^{\infty} \xi\left[-\xi^{2} e^{-\xi z} A+(1-\xi z) \xi e^{-\xi z} B\right. \\
& \left.+\xi^{2} e^{\xi z} C+(1+\xi z) \xi e^{\xi z} D\right] J_{1}(\xi r) d \xi, \\
\sigma_{\theta}= & 2 \mu \int_{0}^{\infty} \xi\left\{\frac{\bar{\sigma}_{r}}{2 \mu}+\frac{\bar{\sigma}_{\theta}}{2 \mu}\right\} J_{0}(\xi r) d \xi-\sigma_{r} \\
= & 2 \mu \int_{0}^{\infty} \xi\left[2 \nu \xi^{2} e^{-\xi z} B+2 \nu \xi^{2} e^{\xi z} D\right] J_{0}(\xi r) d \xi \\
& +\frac{2 \mu}{r} \int_{0}^{\infty} \xi\left[-\xi^{2} e^{-\xi z} A+(1-\xi z) \xi e^{-\xi z} B\right. \\
& \left.+\xi^{2} e^{\xi z} C+(1+\xi z) \xi e^{\xi z} D\right] J_{1}(\xi r) d \xi .
\end{aligned}
$$

Computation of the above equations is generally performed using numerical integration. However, it has always been difficult to maintain good computational accuracy for points in the vicinity of $z=0$. Comparison of the prescribed boundary conditions with the results obtained using DE-integration will show accuracy of DE-integration.

\section{§7. Numerical Integration Using DE-Integration}

\section{§7.1. Axi-symmetric loading}

The external surface loading is as shown in Figure 2. Since a vertical uniformly distributed circular load is considered to act on the surface of a semiinfinite medium, the surface pressure $p$ due to the total load $P$ with radius $a$ may be determined as:

$$
p=\frac{P}{\pi \times a^{2}},
$$

where, $P=49 \mathrm{kN}, a=0.15 \mathrm{~m}$ and $p=0.693 \mathrm{MPa}$. 


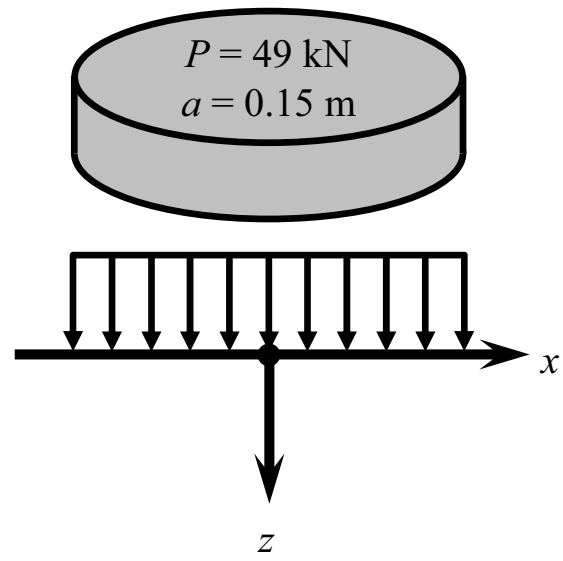

Figure 2. Axi-symmetric Vertical Loading

By using the above loading information $\sigma_{z}$ at the pavement surface was determined using DE-integration and the results are shown in Figure 3. In this figure, square marker $(\square)$ and triangular marker $(\triangle)$ represent results by DEintegration for semi-infinite and finite integrals, respectively and the solid line represents true values. The top figure shows the whole range of computation while the bottom figure shows an enlarged view in the vicinity of the loaded area. Relative error in the DE-integration was set at 1.0E-8.

In this case, the results are supposed to be $0.693 \mathrm{MPa}$ between the $-0.15 \mathrm{~m}$ to $+0.15 \mathrm{~m}$ span, $0.347 \mathrm{MPa}$ at $0.15 \mathrm{~m}$ edge and then zero elsewhere. Results presented in Figure 3 show that for points that are odd multiples of the radius of the load, i.e. $0.15 \mathrm{~m}, 0.45 \mathrm{~m}$ and $0.75 \mathrm{~m}$, semi-infinite DE-integration had problems achieving results with acceptable accuracy. For the case of finite DE-integration, zeros of Bessel functions were used as limits of integration for stepwise computation of the function. However, results by finite DE-integration were poor in the vicinity of $0.15 \mathrm{~m}$. Moreover, since many zeros of Bessel functions were required in order to integrate the function to a good accuracy, the analysis was very time consuming.

An important point to note here is that for values of $z>0$ both semiinfinite and finite DE-integration gave similar results as shown in Figure 4. This figure shows results obtained in the case where $z=0.15 \mathrm{~m}$. True values are not known but it is assumed that since the two integrations procedure achieved similar results, these results may be considered accurate. Computation time in this case was relatively shorter as compared to the case where $z \rightarrow 0$. 

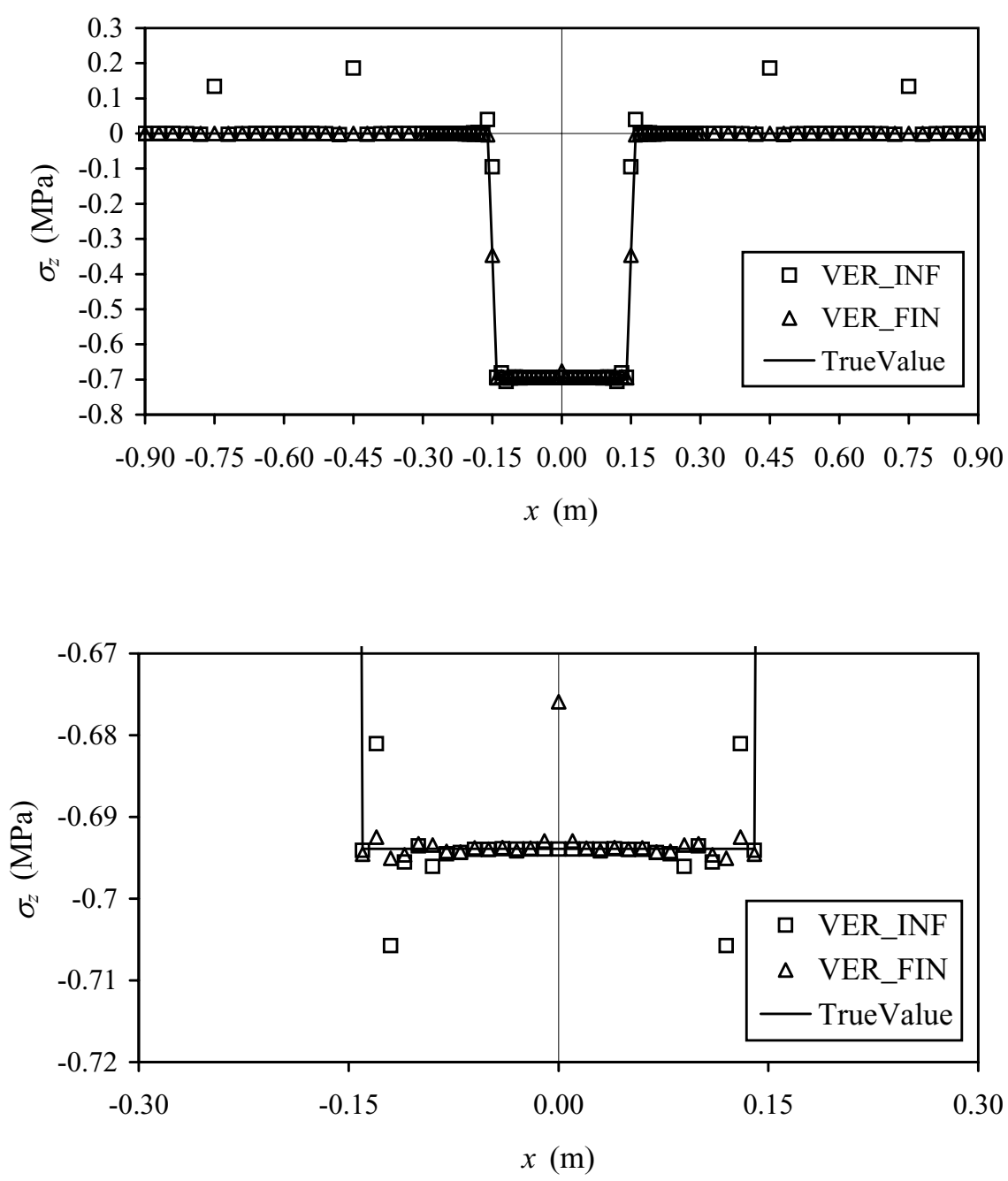

Figure 3. Comparison between DE-integration results and true values Top: Overall view Bottom: Enlarged view near the loaded area 


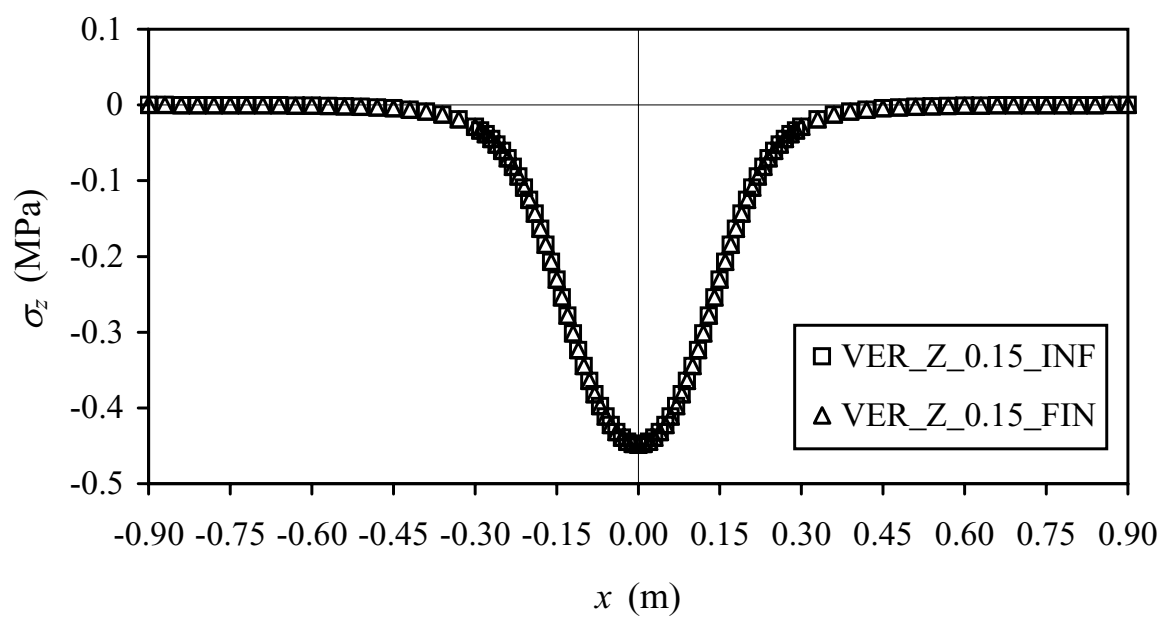

Figure 4. DE-integration results for $z=0.15 \mathrm{~m}$

\section{§7.2. Richardson's extrapolation}

In order to speed up computation time as well as to improve computational accuracy in the vicinity of $z=0$, Richardson's extrapolation was incorporated in the numerical analysis procedure. Furthermore, efficient application of Richardson's extrapolation to the integral may be achieved by the procedure suggested by Prof. Sugihara [6,7]. In this case, the integral

$$
I=\int_{0}^{\infty} f(\xi) d \xi
$$

was modified to:

$$
I=\lim _{b \rightarrow 0} \int_{0}^{\infty} f(\xi) e^{-\xi^{2} b} d \xi
$$

In order to perform Richardson's extrapolation, five values of $b$ were used. They were $b=2^{-n}$ for $n=10,11,12,13,14$. Results obtained using Richardson's extrapolation for both semi-infinite and finite DE-integration are shown in Figure 5. This figure shows a substantial improvement in the accuracy of DE-integration and especially finite DE-integration. The accuracy by semiinfinite DE-integration improved significantly, but there was still a problem in the vicinity of the edge of the load (i.e. $0.15 \mathrm{~m}$ ). 

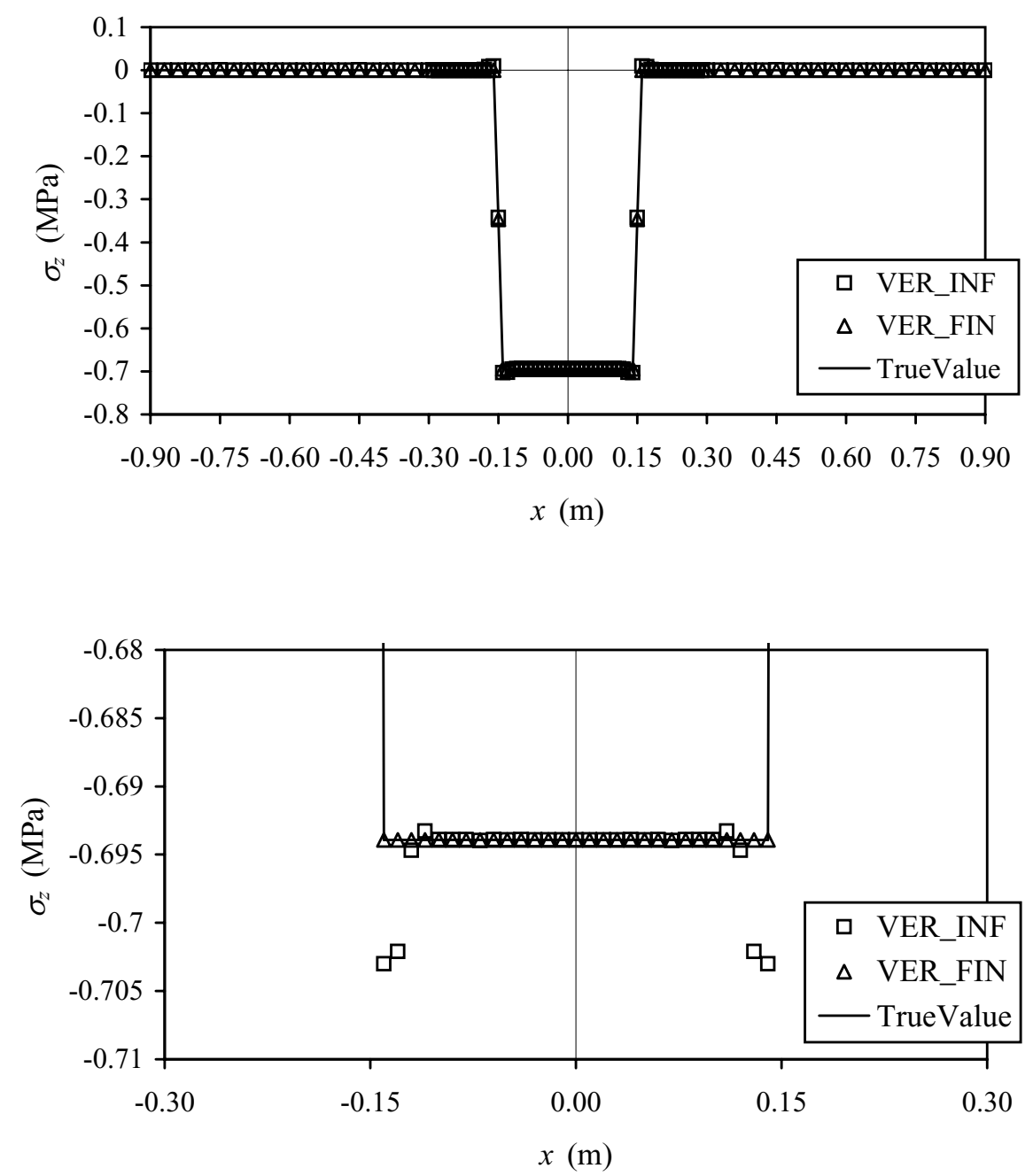

Figure 5. Comparison between DE-integration results with Richardson's extrapolation and true values

Top: Overall view Bottom: Enlarged view near the loaded area 


\section{$\S 8$. General Observations}

Using the stress function and the Hankel transform, a computer code for general analysis of an multi-layered elastic system (GAMES) has successfully been developed. Identification of the problem areas helped in the improvement of the accuracy of computations. Accuracy was improved through the introduction of Richardson's extrapolation in the vicinity of $z=0$. For practical applications, it is recommended that semi-infinite DE-integration be used since the acceptable results for engineering works are obtained in a very short computation time.

The program developed is now capable of performing analysis for up to 10,000 measurement points in an elastic multilayer system with a maximum of 100 layers on the surface of which a maximum of 100 uniformly distributed circular loads act.

\section{References}

[1] Maina, J. W. and Matsui, K., Development of Software for Elastic Analysis of Pavement Structure Due to Vertical and Horizontal Surface Loadings, Journal of Transportation Research Board, No. 1896, TRB, National Research Council, Washington D.C., 2004, pp. 107-118.

[2] Fujinami, K., Maina, J. W. and Matsui, K., Multilayer Elastic Analysis of Pavement Structure due to Torsional Surface Loading, Journal of Materials, Concrete Structures and Pavements, JSCE, No. 781/V-66, Tokyo, Japan, 2005, pp. 171-179.

[3] _ Multilayer elastic analysis of pavement structure due to centripetal surface stress, Journal of Materials, Concrete Structures and Pavements, JSCE, No. 788/V-67, Tokyo, Japan, 2005, pp. 43-51.

[4] Ooura, T. and Mori, M., The double exponential formula for oscillatory functions over the half infinite interval, J. Comput. Appl. Math., 38 (1991), 353-360.

[5] Sneddon, I. N., Fourier Transforms, McGraw-Hill Book Company, 1951.

[6] Mori, M., Developments in the Double Exponential Formulas for Numerical Integration, Proceedings of the International Congress of Mathematicians, (1990), 1585-1594.

[7] Sugihara, M., Methods of numerical integration of oscillatory functions by the DEformula with the Richardson extrapolation, J. Comput. Appl. Math., 17 (1987), 47-68. 